\title{
Adapting UML Activity Diagrams for Mobile Work Process Modelling: Experimental Comparison of Two Notation Alternatives
}

\author{
Sundar Gopalakrishnan, John Krogstie, and Guttorm Sindre \\ Department of Computer and Information Science \\ Norwegian University of Science and Technology (NTNU) \\ Sem Sælands Vei 7-9, 7491 Trondheim, Norway \\ sundar@idi.ntnu.no, krogstie@idi.ntnu.no, \\ guttorm.sindre@idi.ntnu.no
}

\begin{abstract}
Even if geographical aspects such as location is included in several enterprise architecture frameworks [15], enterprise modelling notations seldom capture the "where" aspect, such as the location for performing some activity in a business process. However, for mobile information systems it is often relevant to model where something is supposed to take place. In a previous paper, we suggested some alternatives for small modifications to UML Activity Diagrams to address this, but then only comparing the alternatives analytically. In this paper, we report on a controlled experiment comparing the two most promising notations from the previous paper, one adding location to the activity diagrams by annotations, another indicating location by colour. The experiment investigated both the participants' opinions about the notations and their performance on some tasks requiring understanding of the models. For opinion there was no significant difference, but for task performance there was a significant difference in favour of the notation using colour.
\end{abstract}

Keywords: Requirements specifications, mobile information systems, modelbased development, UML activity diagram, enterprise modeling.

\section{Introduction}

Mainstream process notations used in IS modelling tend to ignore the "where" aspect. For instance, BPMN [1] and UML activity diagrams [2] capture what (objects), how (sequence and parallelism of activities and decisions), who (swimlanes), when (time triggers and time events), and to a very limited extent why (e.g., how a decomposed activity diagram satisfies a higher level activity) - for the latter some extensions with process goals have also been suggested [3] - but not the location of the activities performed. With a view on traditional information systems, where work is performed by people sitting in their offices using desktop computers, the neglect of physical location is understandable - it is much more important whether a task is performed by the purchasing or salary department than whether the worker is sitting in office 221 or 325 . Hence the usage of swimlanes to denote organizational placement rather than geographical placement is easily justified. 
For mobile information systems, however, the location and context of activities performed may be of major importance [4]. Whether a certain task should be performed in the office before going to a client, in the car while driving, after arriving to the client - or possibly any of these places, up to the personal preference of the worker - could have a large impact both on quality, efficiency, worker satisfaction and customer satisfaction, and would therefore be an important process design decision. In turn, this decision would also have a lot of impact on what applications would have to be developed to support the work process, and what requirements that these applications would have to satisfy. For instance, if the task were to be performed while driving a car, this would imply quite different usability requirements than what a desktop application is normally faced with.

It is therefore interesting to look at the possibility of adapting mainstream process notations such as UML activity diagrams to also be able to capture the location of activities. A minor adaptation of a mainstream notation like UML seems more tempting than inventing an entirely new notation because industry is more likely to pick up a notation they are already largely familiar with. Relating to UML, there is also a long tradition in providing modelling profiles of this sort, having small extensions to the core notation. In previous work [5] we presented a number of notation ideas for including location in UML diagrams and compared them analytically using some examples. Some of the notations turned out to be clearly inferior, either having lacking expressive power or economy or becoming messy due to a high number of crossing lines. Two of the proposed notations came out as more promising than the others, one adding location/context by means of annotations (i.e. UML notes) and the other using colour. The rest of the paper is structured as follows: Section 2 presents the result from our prior work and some related work in this area, section 3 discusses the research method, and section 4 presents the experiment results. Section 5 provides analysis and discussion, and section 6 concludes the paper. More insignificant results of this experiment are documented in appendix.

\section{Prior and Related Work}

As already stated in the introduction, two of the proposed notations came out as more promising than the others.

The two alternative notations are shown in Fig 1, both capturing part of the work process within a home care unit, offering practical help and home nursing care to its clients. In the 'Mobile Care'-project, it is planned to better support the mobile aspects of the home care service by providing the employees continuous access to the central health information system (software used in PDA to $\log /$ receive info) and other relevant systems from wherever they are using a combined PC/PDA-solution. This is related to the 'Wireless Trondheim'-project [6], which is currently managing and extending a mobile broadband (WLAN) infrastructure for Trondheim. The shift leader distributes patient visits on available personnel in the morning meeting, each homecare assistant then decides on the sequence of visits to be made while still in the office. Then while driving to the patient's home, the assistant prepares for the visit by obtaining some information about the patient (typically through an audio interface, to 

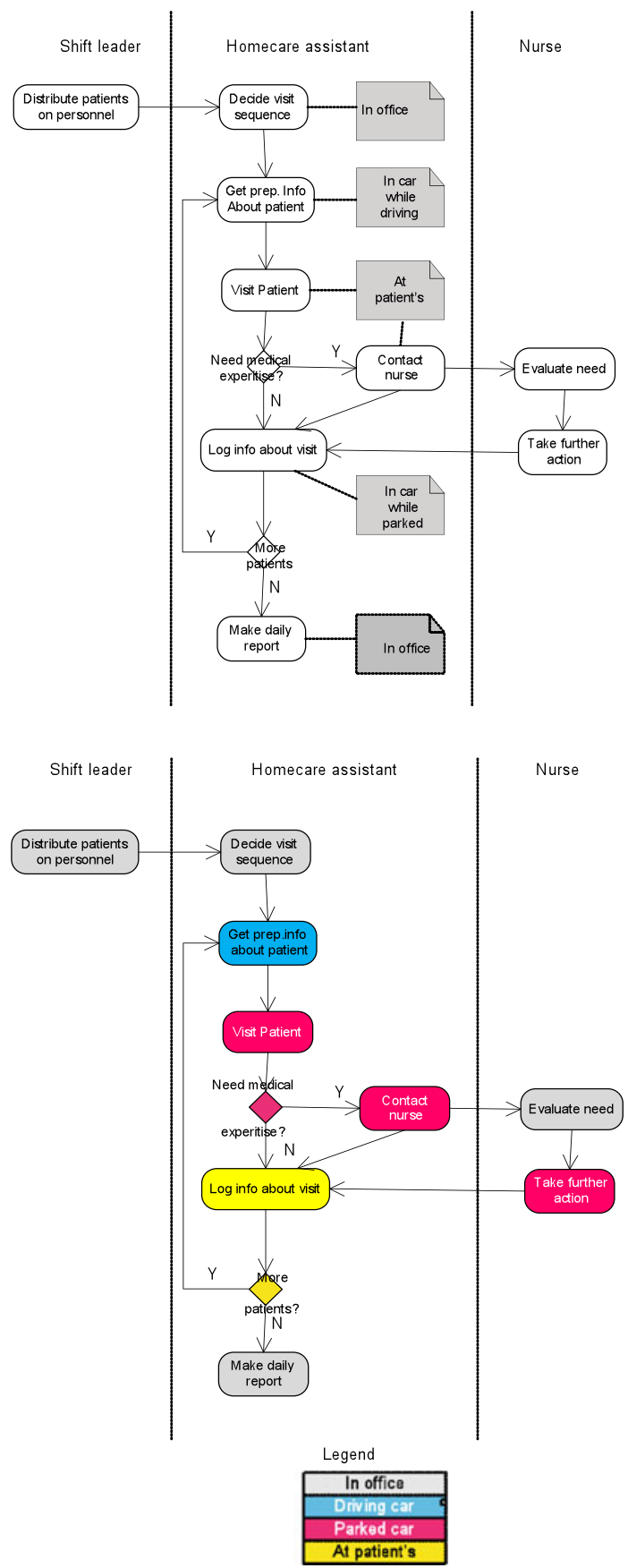

Fig. 1. The two alternative notations - Home Care Case 
be less disruptive for concentrating on the driving). Normally, the patient only needs help with day-to-day activities (e.g., shopping, cleaning, taking the right amount of medication), but in case there are some health complications that the assistant cannot handle, a nurse is contacted. Using a system called Gerica accessible by her PDA, the home care assistant can log information about patients on the go. If the health care assistant needs further medical expertise he/she can request help from the nurse at hospital through logging in information via Gerica. The nurses at the hospital get the request and provide further info/advice to be followed by the healthcare assistant (HCA). Finally the HCA finishes her job by reporting at the office. Locations (e.g., office, car) and context (e.g., parked, driving) are indicated by notes, which are already available in the UML standard [7].

The first notation in the Fig. 1 use notes (in grey) to indicate where each activity is taking place, e.g., in the office, in the car while driving or while parked, or in the patient's home. The second notation in Fig.1 instead uses colour, where office activities have grey notes, activities in the car while driving have blue nodes, while parked red, and in the patient's home yellow. It is easy to observe some pros and cons for each notation. The annotation alternative is a much lesser deviation from standard UML than the colour alternative. The annotations could also have the advantage that the location/context is always quickly found just beside the activity node, while the colour notation requires the user either to remember or consult the colour legend to understand the diagram details. On the other hand, the annotation alternative causes the diagram to have more nodes and links, thus becoming more complex. Another advantage of the colour notation, which is not directly visible in figure 1 , is if one wants to compare several design alternatives putting them side by side (e.g., one where getting info about the patient is done while driving, another where it is done while parked or in the patient's house). Having two such alternatives side by side with the colour notation, it would be immediately spottable where the difference between the two process designs are (because two nodes would be differently coloured while the others are identical), while it would not be so obvious with the annotations, requiring detailed inspection of the text inside the notes. Parts of the outcome of the analytical evaluation in [5] are summarized in Table 1 and 2 below where the alternative notations are compared against traditional UML activity diagrams (but here only including the two above mentioned notations, not some others that were less favourably evaluated). All in all, the analytical evaluation might indicate some advantage for the colour notation. However, as the discussion above reveals this advantage is not entirely clear, and it is impossible to know whether the list of evaluation criteria was complete, and whether all criteria should have equal weight or maybe some are more important than others in determining the practical usefulness of the notations. Hence, such analytical evaluations must be supplemented by empirical evaluation, which is the purpose of the research presented in this paper. A controlled experiment where participants use both notations for the same tasks could be able to reveal whether any notation has advantages when it comes to supporting understanding and problem solving related to the modelled case. Hence such an experiment was performed, and the purpose of the current paper is to report on that experiment. For such experimental study on comparing information modelling methods [19], it is important to show that the two notation alternatives and the cases are informationally equivalent. In other words, we do not want one language to be 
more comprehensive than the other. Since both notations are extensions of the same modelling language, adding the possibility to add location information in two different ways, we can argue for such equivalence.

Table 1. Evaluation of proposed notations with simple and large models

\begin{tabular}{|c|c|c|c|c|c|c|c|c|}
\hline Notation & \multicolumn{2}{|c|}{$\begin{array}{c}\text { Min. deviation from } \\
\text { standard }\end{array}$} & \multicolumn{2}{|c|}{ Expressiveness } & \multicolumn{2}{|c|}{$\begin{array}{c}\text { Intive / Easy to } \\
\text { read }\end{array}$} & \multicolumn{2}{c|}{ ModelComplexity } \\
\cline { 2 - 9 } & Simple & Large & Simple & Large & Simple & Large & Simple & Large \\
\hline Trad. UML & ++ & ++ & -- & -- & ++ & ++ & ++ & ++ \\
\hline Annotated & + & + & + & + & - & -- & + & -- \\
\hline Colours & - & - & ++ & ++ & + & + & ++ & ++ \\
\hline
\end{tabular}

Table 2. Evaluation of proposed notations with SEQUAL framework

\begin{tabular}{|c|c|c|c|}
\hline \multirow{2}{*}{ Notation } & \multicolumn{3}{|c|}{ SEQUAL Framework [8] on Language Quality } \\
\cline { 2 - 4 } & $\begin{array}{c}\text { Organizational } \\
\text { Appropriateness }\end{array}$ & $\begin{array}{c}\text { Domain } \\
\text { Appropriateness }\end{array}$ & $\begin{array}{c}\text { Comprehensibility } \\
\text { Appropriateness }\end{array}$ \\
\hline Traditional UML Act. Diag. & + & + & ++ \\
\hline Annotated & + & + & ++ \\
\hline Colours & ++ & ++ & ++ \\
\hline
\end{tabular}

Walderhaug et al. used UML notations extensively in the MPOWER project [4] with homecare services and conclude that UML profiles [10] can be used as a mechanism for tool chains based on OMG's Model Driven Architecture (MDA) and UML standards [2] [7]. Work on mobile ontologies by Veijalainen [9] supports the idea of the 'where' aspect as essential in mobile processes, but excludes the 'what' aspect. Larsson [10] proposes the three building blocks for knowing the processes list How, What and Why, adds Who for use oriented design approach but omitted the 'Where' concept. Whereas the use of colour is not so common in conceptual modelling, in other 'modelling' areas such as cartography colour is widely used to improve comprehension. Related to comprehension is the work by Bertin [11] on visual variables, where colour is one important differentiator. Moody [12], in his proposal for a 'physics' of notation based his work on among other things the work of Bertin.

\section{Research Method}

Our goal in this paper is to compare the two notations presented above to find out which one gives the best results for the user, in terms of understanding process diagrams or using such diagrams in problem solving activities. Of course, there are a number of ways to make such comparisons, ranging from analytical comparisons, through controlled experiments on small-scale tasks, to usage in large-scale industrial projects. Since our previous work made an analytical comparison, a natural next step 
is to go empirical, i.e. either experiment or industrial case study. Moreover, since the goal here is to compare two notations rather than investigate the merits of one notation, it makes most sense to go for a controlled experiment. With industrial case studies, it would be impossible to do exactly the same project twice; hence any comparison would easily be blurred by a number of confounding factors. With the decision to go for a controlled experiment, the next question is to decide the details about the experimental treatment and the tasks that the participants will perform. The normal "treatment" [13] when experimenting about new diagram notations is to give the participants a quick tutorial about the notation they are going to use, and then supply them with a case and some tasks to solve. For generalizability of the results, a wide variety of tasks might be a good idea, but on the other hand controlled experiments normally have to be constrained to a quite limited time frame - it is hard to recruit enough subjects if it lasts longer than a couple of hours. Hence, we chose to go for two experimental tasks: (i) testing the participants' understanding of the case, measured by their score in answering a number of true/false questions, and (ii) the participants' problem solving abilities, measured by their ability to detect errors in a diagram - this investigated by providing a textual case description declared to be correct and they a diagram assumed to capture this, but deliberately seeded with some errors. In addition we elicited the participant's opinion about the notation through a post-task questionnaire. We thus had three main variables to measure about each notation in the experiment:

Understanding: the participant's fraction of correct answers to the true/false questions.

Error_detection: the participant's fraction of correctly detected errors to the total number of errors in the deficient diagram.

Average_opinion: the participant's average score on 14 questionnaire items about the notation.

Another question is whether to do a within-subjects (i.e., all subjects get both treatments) or between-subjects (half the subjects get one treatment, the other half the other treatment) experimental design. According to [13] a within-subjects design is advantageous if it is possible with the given treatments, since it doubles the sample size and also controls better for selection bias. On the other hand, this design has some added challenges with learning effect, meaning that two different cases must be used and participants divided in four groups in a so-called Latin Squares design, as will be explained below.

Given the abovementioned variables, and since the colour notation came out slightly better than the annotated notation in the analytical comparison in [5], we have the following key hypotheses for our experiment:

H1: the Understanding scores for the colour notation will be better than those for the annotated notation

H2: the Error_detection scores for the colour notation will be better than those for the annotated notation

H3: the Average_opinion scores for the colour notation will be better than those for the annotated notation 
Corresponding null hypotheses could also be formulated, but are not presented here for space concerns. Also, there could be more detailed hypotheses relating to different question groups investigated in the post-task survey, i.e., 5 questions related to Perceived Ease of Use, 5 to Perceived Usefulness, and 4 to Intention to Use. These hypotheses would be similar to H3, i.e., assuming that the colour notation would score better than the annotated notation. Again, these more detailed hypotheses are not shown to save space and found to be less significant.

46 students were recruited from a second year computer science class to take part in the experiment. With the Latin Squares design, these were divided into 4 groups according to which annotation to try first, and on which case the annotation was used, creating 4 different permutations, e.g., annotated first with the home care case, then colour with another case about traffic control, annotated traffic, then colour with home care, colour with home care then annotated traffic, or colour with traffic then annotated home care.

1. The 46 participants were randomly distributed to the four experiment groups in the Latin Squares design. The questionnaire prepared contained four parts, 1. Pre-experiment questionnaire 2. Questionnaire on $\mathrm{CN}$ or $\mathrm{AN}$ with case 1 and post experiment evaluation. 3. Questionnaire on $\mathrm{AN}$ or $\mathrm{CN}$ with case 2 and post-experiment evaluation. 4. Identifying mistakes in $\mathrm{CN}$ and $\mathrm{AN}$ with brief case explanation. The participants performed the following activities during the experiment: Answering a pre-experiment questionnaire: The purpose of the preexperiment questionnaire was simply to investigate the participants' prior knowledge of related topics like UML, process modelling, etc., which can be used to control for any accidental group selection bias in spite of random selection (e.g., one group accidentally containing people with much more relevant experience). This is much less important for a Latin Squares design than for a between-subjects design, but since it only takes a couple of minutes for the participants to answer a few questions about their prior experience with relevant modelling techniques, it still felt worthwhile to do. Questions investigated previous knowledge on modelling, UML, activity diagrams, specifications, IT work experience and knowledge about the domains the cases were taken from (home care and traffic control), in total 8 questions that were to be answered within 5 minutes.

2. Reading a tutorial about the first diagram notation (annotated or colour), using a flight check-in case as an example case description followed by corresponding annotated or colour notation diagram were presented as a tutorial part in the experiment.

3. Being presented with experimental textual case description (home care or traffic control), together with a diagram (annotated or colour), and at the end of this, participants must answer 12 true/false questions related to that particular case.

4. Answering a post-task questionnaire about the notation just used, containing 14 questions investigating Perceived Ease of Use (PEOU), Perceived Usefulness (PU), and Intention to Use (ITU) as inspired by the TAM model [14].

5. Repeating steps $2-4$ using the other notation on a different case. Totally 54 minutes were allotted to complete steps 2-5 and return the booklet. 
6. A separate booklet with textual description and notations on both cases deliberately seeded with some errors was distributed again and now the task was to find all the errors in the diagram (i.e., discrepancies between the diagram and the case description) i.e., all the students had questionnaire on both notations and both cases in steps 2-5 and also in 6. Of course, the seeded errors (5 errors per notation) were the same both for the annotated and coloured variants of the diagrams. The allotted time to complete these tasks was 10 minutes. The experiment performed in Latin squares design and the cases distribution is as shown in table 3 .

Table 3. Latin square experiment questionnaire distribution to student groups

\begin{tabular}{|l|l|l|}
\hline Group Id & $\begin{array}{l}\text { (Understanding+ TAM } \\
\text { factor) Questionnaire on }\end{array}$ & $\begin{array}{l}\text { Error Identification } \\
\text { Questionnaire on }\end{array}$ \\
\hline Group A & $\begin{array}{l}\text { Annotated Home Care }+ \\
\text { Colour Traffic Control }\end{array}$ & $\begin{array}{l}\text { Annotated traffic Control } \\
+ \text { Colour Home Care }\end{array}$ \\
\hline Group B & $\begin{array}{l}\text { Colour Traffic Control }+ \\
\text { Annotated Home Care }\end{array}$ & $\begin{array}{l}\text { Colour Home Care }+ \\
\text { Annotated traffic Control }\end{array}$ \\
\hline Group C & $\begin{array}{l}\text { Annotated traffic Control }+ \\
\text { Colour Home Care }\end{array}$ & $\begin{array}{l}\text { Annotated Home Care }+ \\
\text { Colour Traffic Control }\end{array}$ \\
\hline Group D & $\begin{array}{l}\text { Colour Home Care }+ \\
\text { Annotated traffic Control }\end{array}$ & $\begin{array}{l}\text { Colour Traffic Control }+ \\
\text { Annotated Home Care }\end{array}$ \\
\hline
\end{tabular}

\section{Experiment Results}

The results for the performance of the participants on the tasks of understanding (answering 12 true/false questions) and problem solving (detecting errors in diagrams relative to a natural language case description) are summarized in table 4 . As can be seen, both variables turned out with a significant advantage in favour of the coloured notation, with effect sizes of 0.51 for understanding and 0.45 for error detection, which are both small to moderate effects according to [16]. For the error detection task, there were however 3 students who performed extremely poorly compared to the others, one found zero errors with both notations, one found zero errors with colours and only 1 with annotations, and another found 1 with annotations and 2 with colours. Assuming that these participants might not have done their best with the task, they might be treated as outliers and excluded from the analysis, but the result $(\mathrm{N}=43)$ still gives a significant advantage in favour of colours, now with an effect size of 0,77 which is a moderate effect.

The results for the participants opinions about the two notations, as indicated by their answers to the TAM-inspired post-task questionnaire, is shown in Table 5. As indicated there was a slight advantage for the coloured notation for PEOU and ITU, but not for PU, and no differences were significant, neither for the overall average nor for the single TAM factors. 
Table 4. Comparison of performances with the two notations

\begin{tabular}{|l|l|l|l|l|l|l|l|}
\hline $\begin{array}{l}\text { Compared } \\
\text { variable } \\
\text { (N=46) }\end{array}$ & $\begin{array}{l}\text { Coloured } \\
\text { diagram }\end{array}$ & \multicolumn{2}{|l|}{$\begin{array}{l}\text { Annotated } \\
\text { diagram }\end{array}$} & Difference & $\begin{array}{l}\text { Effect } \\
\text { Size }\end{array}$ & $\begin{array}{l}\text { Sign.? Y/N } \\
\text { (p-value) }\end{array}$ \\
\cline { 2 - 7 } Mean & SD & Mean & SD & & & \\
\hline Understanding & 0.960 & 0.057 & 0.926 & 0.078 & 0.0344 & 0.51 & $Y(0.01)$ \\
\hline Error detection & 4.50 & 1.34 & 3.93 & 1.17 & 0.57 & 0.45 & $Y(0.001)$ \\
\hline $\begin{array}{l}\text { Errors (w/o } \\
\text { outliers, N=43) }\end{array}$ & 4.77 & 0.53 & 4.16 & 1.04 & 0.60 & 0.77 & $Y(0.001)$ \\
\hline
\end{tabular}

Table 5. Comparison of TAM factors with the two notations

\begin{tabular}{|l|l|l|l|l|l|l|}
\hline $\begin{array}{l}\text { Compared } \\
\text { variable } \\
\text { (N=46) }\end{array}$ & \multicolumn{2}{l|l}{$\begin{array}{l}\text { Coloured } \\
\text { diagram }\end{array}$} & \multicolumn{2}{l|}{$\begin{array}{l}\text { Annotated } \\
\text { diagram }\end{array}$} & Difference & $\begin{array}{l}\text { Significant? } \\
\text { Y/N } \\
\text { (p-value) }\end{array}$ \\
\cline { 2 - 5 } & Mean & SD & Mean & SD & & \\
\hline PEOU & 3.97 & 0.63 & 3.86 & 0.51 & 0.11 & $\mathrm{~N}(0.2)$ \\
\hline PU & 3.95 & 0.79 & 3.96 & 0.67 & 0.01 & $\mathrm{~N}(0.12)$ \\
\hline ITU & 3.49 & 0.89 & 3.26 & 0.83 & 0.23 & $\mathrm{~N}(0.27)$ \\
\hline $\begin{array}{l}\text { Average_ } \\
\text { opinion }\end{array}$ & 3.83 & 0.67 & 3.73 & 0.54 & 0.1 & $\mathrm{~N}(0.16)$ \\
\hline
\end{tabular}

All in all, then, we have the following situation for our hypotheses:

- H1 was confirmed; there was indeed a significant advantage for the coloured notation when it came to the measured understanding in terms of the scores for the true/false questions.

- H2 was also confirmed; there was a significant advantage for the coloured notation when it came to measured problem solving capability in terms of the number of identified errors in the diagrams.

- H3 must be rejected, as no significant advantage could be found for any notation when it came to responses to the post-task questionnaire investigating the participants' opinions about the notations and these data are documented in appendix.

Normally, one might think that the modelling technique that gives the best performance would also get the best evaluation from the participants when asking about their opinion. However, the situation that performance advantages has not necessarily yielded a similar advantage in opinion has also been observed in other experiments, for instance in [17] where one technique had a significant performance advantage, yet no such advantage was found for opinion, indeed there was no notable correlation between the participants' performance with a technique and opinion about that technique. 


\section{Threats to Validity}

Wohlin [18] suggests four relevant categories for discussing threats to validity in experiments: conclusion validity, construct validity, internal validity and external validity. Conclusion validity concerns the relationship between the treatment given and the outcome in measured variables. One important question is whether the sample size is big enough to justify the conclusions drawn, which can be investigated by means of the calculated effect size (ES). We accepted two hypotheses, one about better understanding with the coloured notation $(\mathrm{ES}=0,51)$ and one about better error detection ( $E S=0,45$, or with outliers removed, $E S=0,77)$. Denoting the Type I error probability by $\alpha$ (accepting a relationship which really is not there) and the Type II error probability by $\beta$ (overlooking a relationship that really was there), the following holds:

$$
N=\frac{4\left(u_{\alpha / 2}+u_{\beta}\right)^{2}}{E S^{2}} .
$$

If we use $\alpha=0.05$ (our threshold for accepting a relationship as significant) and $\beta=$ 0.20 , we get $\mathrm{N}=32 /(\mathrm{ES})^{2}[16]$ as a required sample size. This means that we should have had a sample size of 123 for understanding and 158 for error detection (or 54 for the ES achieved when outliers were removed). Our sample size was only 46 (or 43 with outliers removed). The fact that our sample size is smaller than the required ones means that our results have to be interpreted with caution, although the difference clearly came out as significant.

Construct validity is concerned with the inference from the measures made in the experiment to the theoretical constructs we were trying to observe (understanding, problem solving effectiveness). Of course, there are other ways to explore understanding than true/false questions after looking at a case description and diagram, and other ways to explore effectiveness than asking participants to identify errors. But at least, identification of errors is an important task in system development (for instance in connection with reviews / QA), and answering questions is at least one relevant way of testing understanding. Given the limited type (only true/false) and nature of the questions, it must still be admitted that they will not measure every aspect of understanding, and that more experiments with a wider range of experimental tasks would be necessary to draw more certain conclusions.

Internal validity means that the observed outcomes were due to the treatment, not to other factors. Our Latin-Squares experimental design was used to eliminate selection bias, and to control for any learning effects or effects of which case was used with which technique. In addition we performed a pre-experiment questionnaire to test whether other factors such as previous relevant experience could explain the differences between the various groups, but not finding any such effects.

External validity is concerned with the question of whether it is possible to generalize from the experimental setting to other situations, most importantly to industrial systems development. The use of students instead of practitioners is a notable threat. However, this threat is reduced by the fact that we are only trying to compare two notations in relative terms, not evaluate their merits in more absolute terms. Moreover, these adapted notations would be new also to practitioners, thus 
reducing the advantage they might otherwise have had over students (e.g., if the practitioners were familiar with the notations from before and had used them a lot at work).

\section{Conclusion}

For the development of mobile and multi-channel information systems it might be important to have process notations able to capture the "where" aspect, i.e., the location or context of the various activities. For instance it might have a substantial impact on usability requirements if an activity is supposed to be performed in office with a desktop PC or using some mobile device while driving a car. Hence, we have suggested some alternative adaptations of the UML Activity Diagram notation to enable the capturing of location and context. In this paper, we have reported on a controlled experiment comparing two such adapted notations, one using annotation and the other using colour to capture the location/context of activities. In a previous analytical evaluation, the colour alternative came out as slightly better, hence we hypothesized an advantage for the colour notation for all the variables we measured, i.e., that the colour notation would prove better both for understanding, error detection and participant opinion about the notations. Statistical analyses confirmed an advantage for understanding and error detection, but not for the opinion.

The effect sizes, however, were not large enough to support conclusion validity. A natural first step for further work would therefore be to perform the same or similar experiments with more subjects. It might also be interesting to modify the experimental tasks. The case descriptions and corresponding activity diagrams were fairly small and simple, and more complex cases would make future experiments more representative of realistic industrial enterprise modelling task. More complex tasks could also bring the advantage of larger effect sizes - if indeed one notation has an advantage over the other. In particular, the understanding task with true and false questions seems to have been fairly easy, the average score being above $90 \%$ with both notations, which means that any difference will necessarily be small.

Other venues for future work could be to include yet other notations in similar experiments. While colour came out as the best in this experiment, it does have problems w.r.t. colour-blind users. Hence, it could be interesting to see if a notation using pattern fills (also mentioned as a possible visual variable by Bertin) in the activity nodes could do just as well as colour.

Finally, of course, experiments should be supplemented with larger case studies in enterprise modelling / systems analysis projects, preferably in industry. This would provide a better indication whether any advantages observed in a limited experimental setting also hold for real world usage.

\section{References}

[1] Unified Modelling Language, http : / /www . uml . org (accessed 4.6.2010)

[2] Business Process Modelling Notation, http : / /www . bpmn . org / (accessed 4.6.2010) 
[3] Korherr, B., List, B.: Extending the UML 2 Activity Diagram with Business Process Goals and Performance Measures and the Mapping to BPEL. In: Roddick, J., Benjamins, V.R., Si-said Cherfi, S., Chiang, R., Claramunt, C., Elmasri, R.A., Grandi, F., Han, H., Hepp, M., Lytras, M.D., Mišić, V.B., Poels, G., Song, I.-Y., Trujillo, J., Vangenot, C. (eds.) ER Workshops 2006. LNCS, vol. 4231, pp. 7-18. Springer, Heidelberg (2006)

[4] Walderhaug, S., Stav, E., Marius Mikalsen, M.: Experiences from Model-Driven Development of Homecare Services: UML Profiles and Domain Models. In: Chaudron, M.R.V. (ed.) Models in Software Engineering. LNCS, vol. 5421, pp. 199-212. Springer, Heidelberg (2009)

[5] Gopalakrishnan, S., Sindre, G.: Alternative Process Notations for Mobile Information Systems. In: Proc. I-ESA 2010, Coventry, UK, Springer, Heidelberg (2010)

[6] Andresen, S., Krogstie, J., Jelle, T.: Lab and Research Activities in Wireless Trondheim. In: Proceedings of IEEE International Symposium on Wireless Communication Systems, pp. 385-389. IEEE Computer Society, Los Alamitos (2007)

[7] Booch, G., Rumbaugh, J., Jacobson, I.: The Unified Modelling Language: User Guide. Addison-Wesley, Reading (1999)

[8] Lillehagen, F., Krogstie, J.: Active Knowledge Modelling of Enterprises. Springer, Heidelberg (2008)

[9] Veijalainen, J.: Developing Mobile Ontologies; who, why, where, and how? In: International Conference on Mobile Data Management, Manheim, Germany, pp. 398401. IEEE, Los Alamitos (2007)

[10] Larsson, A. V.: Designing for use in a future Context - Five Case Studies in Retrospect, PhD thesis No: 1034, Institute of Tech., Linkoping Univ., Sweden (2003)

[11] Bertin, J.: Semiology of Graphics: Diagrams, Networks, Maps. University of Wisconsin Press (1983)

[12] Moody, D.L.: The "Physics" of Notations: Towards a Scientific Basis for Constructing Visual Notations in Software Engineering. IEEE Transanctions on Software Eng. 35(6), 776-779 (2009)

[13] Field, A., Hole, G.: How to Design and Report Experiments. Sage Publications, London (2003)

[14] Davis, F.D.: Perceived usefulness, perceived ease of use and user acceptance of information technology. MIS Quarterly 13, 319-340 (1989)

[15] Zachman, J.A.: A framework for information systems architecture. IBM Systems Journal 26(3), 276-291 (1987)

[16] Hopkins, W.G.: A New View of Statistics. University of Queensland, Australia, Brisbane (2001)

[17] Opdahl, A.L., Sindre, G.: Experimental comparison of attack trees and misuse cases for security threat identification. Information and Software Technology 51(5), 916-932 (2009)

[18] Wohlin, C., Runeson, P., Höst, M., Ohlsson, M.C., Regnell, B., Wesslén, A.: Experimentation in Software Engineering: An Introduction. Kluwer Academic, Norwell (2000)

[19] Siau, K.: Informational and Computational Equivalence in Comparing Information Modeling Methods. Journal of Database Management 15(1), 73-86 (2004) 


\section{Appendix}

In this section, some of the additional results, questionnaire information and detailed description of the other case (police traffic control) of this student experiment are documented. This below section will give the reader a bird's eye view on how the experiment has been performed too.

\section{A1 Police Traffic Control Case}

The UML diagram notations presented in this study is based on a simple task in the complete police traffic control case. In this study we used alternate notation Annotated Notation (AN) in view with mobile information systems. Before getting into the case, it may be useful to know the background information about the case, how the traffic police utilising mobile devices for their work. The leader of the police allots control locations for each police assistant personnel for controlling the vehicles through those locations. Controlling includes following up things such as driver license, speeding, drunken driver...etc. Police Assistant Personnel (PAP) receives info

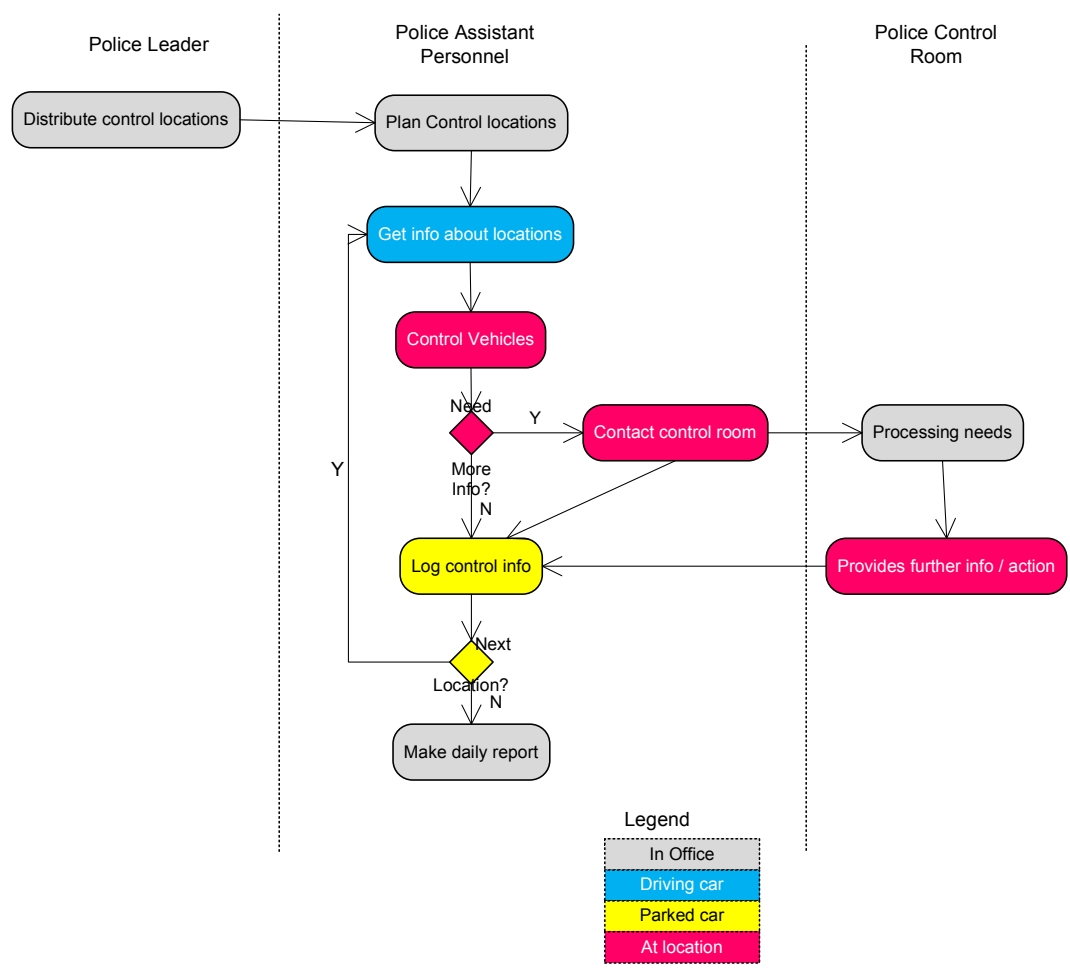

Fig. 2. Police traffic control case- Color Notation $(\mathrm{CN})$ 
about control locations at the office from leader. PAP plans and gets info about the control locations while driving the car. After reaching the control location, PAP controlls the drivers and vehicles. If he decides he need more info/personnel he contacts through mobile/radio/hand held devices...etc the Police control room. The control room provides necessary info/further actions to PAP at control locations. PAP completes the scheduled task for scheduled hour at particular locations and logs all the info from a parked car. He repeats the task until PAP finishes all control locations for the day. After completion, PAP returns to the office and make a daily report. Fig. 2 is based on Color Notation $(\mathrm{CN})$ and Fig. 3 Annotated Notation (AN) of UML activity diagram reflecting the police traffic control case.

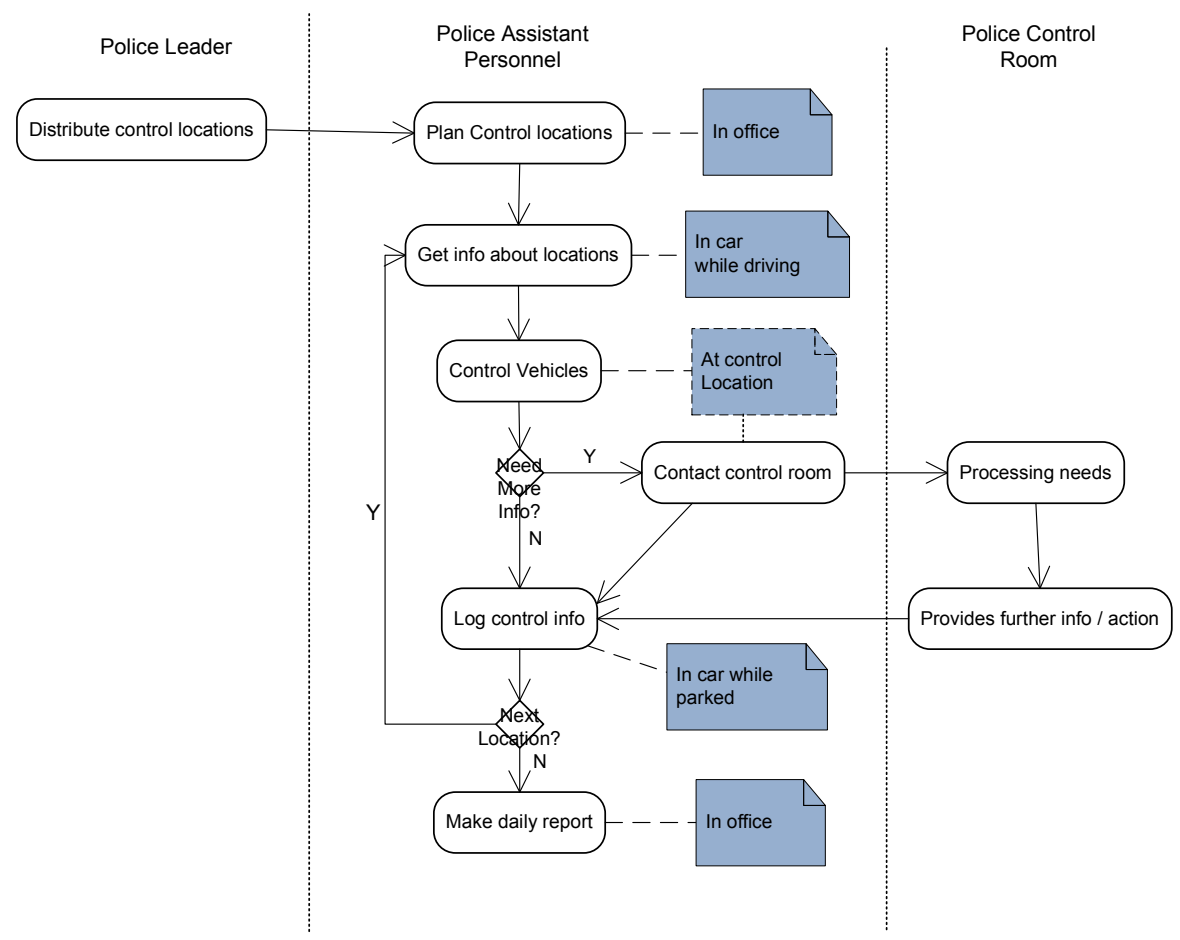

Fig. 3. Police traffic control case -Annotated Notation (AN)

\section{A2 Questions about Home Care Case with Annotated Notation}

(Please write down whether statements are True/False)

1. Home Care Assistant (HCA) receives the patient list to be visited at the office.

2. HCA has been given the patient list along with visit sequence.

3. HCA contacts the nurse while driving the car usually. 
4. If HCA needs medical experts suggestion HCA returns to office to meet Leader.

5. All the patients visit in the list visits HCA at the hospital.

6. HCA collects all preparatory info about patients at the office itself.

7. Patients visited in sequence as per patient's desire.

8. HCA visits all the patients in the list at the patients place.

9. HCA gets the nurse/experts suggestion while driving car.

10. Nurse comes down to patient's location and provide further info if it is needed by HCA.

11. HCA returns to office after visiting one patient in the list to log info about visit.

12. HCA starts and completes his/her duty at office.

\section{A3 Questions about Police Traffic Control Case with Color Notation}

(Please write down whether statements are True/False)

1. Police Leader (PL) visits all control locations along with Police Assistant Personnel (PAP).

2. PAP starts his duty from office everyday.

3. Always PAP gets the control locations distributed by police Leader (PL).

4. After controlling vehicles at each location PAP return to office to log info.

5. If PAP requires more info during controls from control location, he/she contacts police control room.

6. PAP usually prepares daily report at control locations.

7. PAP logs info after each control location visit in parked car.

8. Daily report was made each day by PAP at office.

9. PAP must contact control room before each control location visit.

10. Police Leader prepares daily report for PAP at office.

11. PAP contact Police Leader if PAP needs more info from control locations.

12. PAP returns to office only after completing all scheduled control locations visit and makes daily report at office.

\section{A4 Pre-experiment Analysis}

Based on the results of questionnaires A2 and A3, it is found that colour specification marginally influence on understanding the context of the cases. The below Fig. 4 shows reflects the same. 


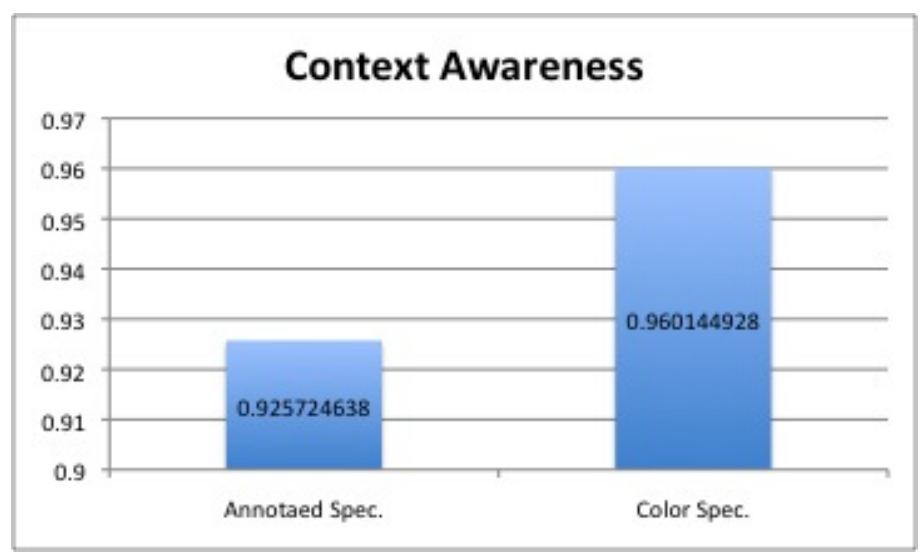

Fig. 4. Understanding (Context Awareness) of case definition through Notations

\section{A5 Post Experiment Questionnaire and Results}

The TAM model was used with the three factors Perceived ease of use (PEOU), Perceived usefulness (PU) and Intention to use (IU). As a part of this evaluation fourteen questions put for the students to answer based on their experience on using these two notations. On evaluating these experiences it is found that the colour notations outperformed insignificantly the annotated notation in all three PEOU, PU and IU aspects. Post-experiment questionnaire with 14 questions with results are Fig. 5 and in table 6.

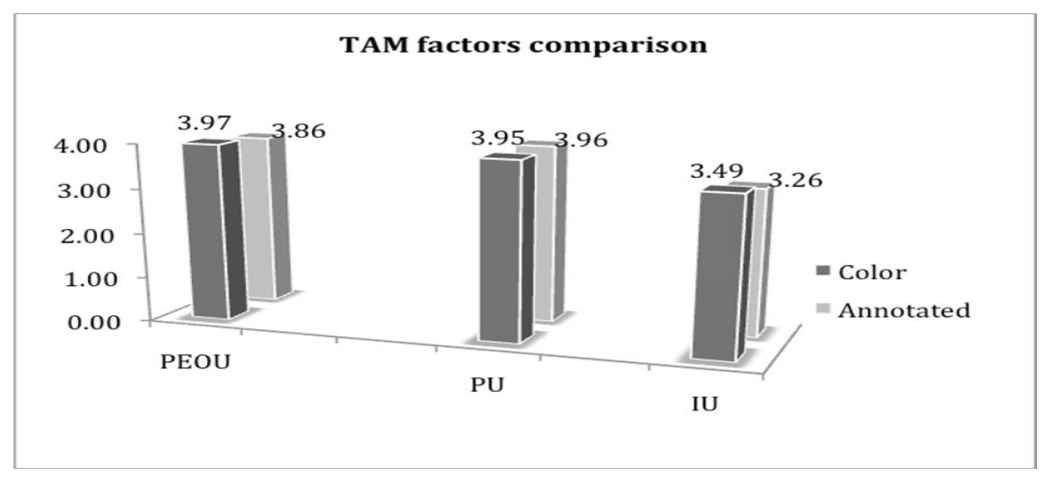

Fig. 5. Post-experiment TAM Questions analysis 
Table 6. Result with TAM questionnaire on both notations

\begin{tabular}{|c|c|c|c|c|}
\hline $\begin{array}{l}\text { Question } \\
\text { id. }\end{array}$ & TAM Questionnaire & $\begin{array}{l}\text { Related } \\
\text { TAM } \\
\text { factor }\end{array}$ & $\begin{array}{l}\text { Annotated } \\
\text { Notation } \\
\text { (max.avg. } \\
\text { value :5) }\end{array}$ & $\begin{array}{l}\text { Colour } \\
\text { Notation } \\
\text { (max.avg. } \\
\text { value :5) }\end{array}$ \\
\hline 1 & $\begin{array}{l}\text { Notation gave me a better understanding } \\
\text { of the activity where it is performed }\end{array}$ & PEOU & 4.435 & 4.652 \\
\hline 2 & $\begin{array}{l}\text { It would be easy to get used to the } \\
\text { Notation in a project. }\end{array}$ & & 4.217 & 4.239 \\
\hline 3 & $\begin{array}{l}\text { I found this Notation is very easy tof } \\
\text { master. }\end{array}$ & PEOU & 4.000 & 4.087 \\
\hline 4 & $\begin{array}{l}\text { If I need to identify where the activityl } \\
\text { process done in a future project, I would } \\
\text { use this Notation }\end{array}$ & & 3.130 & 3.435 \\
\hline 5 & $\begin{array}{l}\text { I would have found where the activities } \\
\text { has been performed by using common } \\
\text { sense }\end{array}$ & PEOU & 3.0434 & 3.174 \\
\hline 6 & $\begin{array}{l}\text { I found not hard to use and recognize this } \\
\text { notation }\end{array}$ & PEOU & 3.978 & 4.109 \\
\hline 7 & $\begin{array}{l}\text { I was not often confused about how tof } \\
\text { apply this Notation to the problem } \\
\text { (activity diagrams) }\end{array}$ & PEOU & 3.848 & 3.848 \\
\hline 8 & $\begin{array}{l}\text { This Notation made the activity diagrams } \\
\text { more systematic. }\end{array}$ & & 3.848 & 3.978 \\
\hline 9 & $\begin{array}{l}\text { If I am employed in a company in future } \\
\text { discusses what technique to introduce for } \\
\text { where the activities performed argue and } \\
\text { someone suggest this Notation, I would } \\
\text { support that }\end{array}$ & & 3.348 & 3.696 \\
\hline 10 & $\begin{array}{l}\text { I can read this Notation quickly, I } \\
\text { understand the notation diagram }\end{array}$ & $\mathrm{PU}$ & 3.957 & 3.870 \\
\hline 11 & This Notation will be easy to remember & $\mathrm{PU}$ & 3.891 & 3.891 \\
\hline 12 & $\begin{array}{l}\text { This Notation made me more productive } \\
\text { in finding where the activities been } \\
\text { performed. }\end{array}$ & & 3.891 & 3.761 \\
\hline 13 & $\begin{array}{l}\text { I will try this notation if I been assigned I } \\
\text { in my future work involving mobile } \\
\text { process }\end{array}$ & & 3.239 & 3.478 \\
\hline 14 & $\begin{array}{l}\text { If I am working as a freelance consultant I } \\
\text { for a customer who needs help finding } \\
\text { where the activities (mobile processes) } \\
\text { performed to his project, I would use AN } \\
\text { in discussions with that customer. }\end{array}$ & & 3.326 & 3.348 \\
\hline
\end{tabular}

